\title{
Physical and Psychological Factors Inhibiting the Use of Technological Devices in the Development of Collaborative Feedback in the Reflective Practice amongst Trainee Teachers
}

\author{
Gordon Ade-Ojo \\ University of Greenwich, School of Education and academic associate of the University \\ of South Africa (UNISA) \\ Nicola Sowe \\ University of Greenwich, School of Education
}

\section{Background}

Much importance has been attached to the promotion and development of a collaborative form of feedback in the context of reflective practice. Two main conclusions from relevant studies are that collaborative feedback contributes to the development of reflective practice, and that the predominant structure of feedback practice has not engendered the development of collaborative feedback (Copland 2008, Copland, Ma and Mann 2009 and 2010, Edge 2005, Alexander 2005, Ade-Ojo and Sowe, 2011). Responses to these conclusions have varied. While some studies have explored the underpinning drivers of feedback in reflective practice (Copland 2008, Edge 2005), others have looked at the processes and physicality of the structures that can support the development of a collaborative approach to feedback (Sowe and Ade-Ojo 2011, Mula 2009, Dyke, Harding and Lajeunesse, 2006). With the latter, one of the more common areas that has been explored is the use of technological devices such as video and digital recordings.

There is some consensus that the introduction of these devices contribute to the evolution of effective and collaborative feedback in reflective practice development of trainees (Research for Teachers 2007) and that, video-based recording offers student teachers to undertake quality self reflection (Robinson and Kelly, 2007, Dymond and Bentz, 2006, Sherin and van Es, 2005, Kong, Shroff and Hong, 2009:546). Furthermore, some studies have sought to firmly link the use of these technological devices to feedback as an element of reflective practice. For example, Dyke, Harding and Lajeunesse (2006) conclude that 
synchronous digital video was favourable in the delivery of feedback just as Sowe and Ade-Ojo (2011) confirmed that digital recording was highly useful in the development of non-didactic feedback. However, many studies have explored what they consider to be potential and real draw-backs of the use of these devices. For example, Mula (2009) describes 'the agony and the ecstasy' of a digital teaching and learning environment, Research for Teachers (2007) highlights trainees feelings 'of being exposed to risk' (p.1), while Halter (2004) draws our attention to the fact that 'many questions about their \{Digital video\} use and impact remain to be explored and answered' while at the same time advocating the need to focus on both 'the technical aspect of technology and digital video' and 'the human aspects of reflection and collaboration using digital media' (p.2).

In many of these studies, however, much attention appears to have been focused on the technical aspects with little attention paid to how technology contributes to and promotes collaborative feedback as a component of self-reflection. Mula (2009) dwells extensively on the cognitive barriers to technology and 'the hurdle of complexity' arguing that, 'you can have the best technology, the most advanced functionality possible, but if people don't want it- perhaps because they aren't equipped to use it- then it is in danger of falling flat', and that, 'as technology continues to advance and universities look to take advantage of them to respond to the dynamic changing nature of learning and their learners' desire to learn in their time and space, we need to find ways to overcome the hurdle of complexity'(p.21). The central message emanating from these studies suggests that the focus of exploration remains on processes and technicality and not on the actual practice and potential outcomes of practice. The result is that the crucial element of how trainees relate to the feedback component as an element of their own development is somehow hidden and perhaps lost. Crucial questions like what emotional and psychological factors could inhibit trainees' engagement with these devices in the course of developing their self reflection ability becomes inevitably forgotten in the desire to explore the obvious technical inhibitions. It is in response to the potential loss of this rather important factor that this study, which is a part of a larger study, sought to explore not only the physical but also the less obvious emotional and psychological inhibitions.

The larger study was built around the exploration of the role and impact of the use of technological devices such as digital recording in facilitating a less-autocratic regime of evaluative post-observation feedback. The various elements of the research sought the views of both trainees and tutors on a teacher training programme. While one element of the research focused on the positive impact of using digital recordings, another sought the opinions of tutors on how it has helped them to develop a more collaborative regime of evaluative feedback. A third focus of the study was on generating a framework for providing what the researchers call co-productive feedback (Ade-Ojo and Sowe 2012, in press). The final focus, which is what is reported in this paper focuses on potential physical and psychological inhibitions to the use of digital recordings from the view point of trainee teachers.

In effect, the central research questions that this paper aimed to answer were as follows. First, what are the factors that might inhibit the achievement of a collaborative evaluative feedback when facilitated by the use of digital recording? Second, do trainee teachers have a difference in perception between these potential problems in terms of whether they are psychological or physical in origin? Ultimately, the goal for us is to set the scene for ameliorating the negative factors that might inhibit trainees and their trainers from taking advantage of the huge underlying strengths that the use of technological devices can provide. 


\section{A conceptual framework for the role of feedback in evaluation}

Feedback as an element of teacher evaluation is often perceived from two functional perspectives: improvement and accountability (OECD 2009). While with the former, the goal is 'to improve the teacher's own practice by identifying strengths and weaknesses for further professional development, with the latter, the focus is to 'hold teachers accountable for their performance, associating it to a range of consequences for their career' (OECD, 2009: 8-9). Given the varying focus of these two concepts of the role of evaluation and by implication, its components such as feedback, it becomes inevitable that there will be tension between the demands of these two. One consequence of this is that most evaluative models utilise what Stronge and Tucker (2003) present as a combination of models for many purposes and methodologies leading inevitably to some form of conflict as indicated in Avalos and Asaael (2006) cited in OECD (2009:9); 'An emphasis on accountability may in some instances lead teachers to feel insecure or fearful and reduce their appreciation of their work. By contrast, teachers and their unions expect opportunities of social recognition of their work and opportunities for professional growth through the development of a formative system of teacher evaluation'. Because of this tension, trainee teachers may naturally experience inhibitions which originate not only from the well documented physical sources but also from psychological sources.

\section{Research implementation and data collection processes}

The implementation process was initiated with the negotiation of consent with the trainees with assurance of anonymity and confidentiality (Crow and Wiles 2008). Consent was also gained from the trainees' employers and learners. A template for an evaluative framework was offered that supported the trainee's self evaluation. The final phase of the process which involved a focus group discussion was also negotiated and agreed. Underpinning the entirety of the implementation process is the recognition of the relationship between researcher and the researched. Given that the researchers were tutors of the participants, participation was strictly on a voluntary basis thus reducing researcher intimidation (Hobbs and Kubanyiova 2008).

\section{Data collection and analysis}

The research was aligned to a mixed-method approach (Cresswell 2003). Data was collected through a number of methods including a questionnaire, a focus group and a tutor discussion/interview thus promoting triangulation (Bell 2010, Coghlan and Brannick 2010). While the data collected through the use of a questionnaire offered the opportunity to present patterns of distribution and frequency of occurrences, the data collected through the use of the other two methods facilitated engagement with the individual and collective reasoning behind what was presented in the quantitative form. Both open and closed questions were used with a thoughtful sequencing to provide a richness of data whilst easily allowing for comparative analysis.

In analysing the quantitative data collected through the use of a questionnaire, the SPSS database spreadsheet was used to map out the frequency of occurrence and distributional patterns emerging from participants' responses. Central to this method of analysis was a process of recording, transcription and codification. The element of codification was facilitated through a simple system of semantic denotation and connotation. These codified responses enabled us to feed in responses to the SPSS spreadsheet leading to the exploration of a number of functions in order to capture information about distribution and 
frequency of occurrence.

The collection and analysis of the second set of data draws from the principle of linguistic ethnography (Copland, 2010, Creese, 2008, Tusting and Maybin, 2007) which is built on a framework that attempts to integrate the tools of ethnography with elements of linguistic data collection and analysis (Rampton 2007). This promoted the foregrounding of 'a reflexive approach' (Copland 2010:467). Prior to the collection of the focus group interview data, an initial analysis of the questionnaire data was carried out. This provided the guidelines which served as prompts for the direction of some of the discussions. The goal of the focus group was to validate the questionnaire findings and to stimulate participants to make explicit their views, perceptions and motives (Punch, 2003). The unstructured nature of the discussion allowed a conversation to explore personal experience and feelings in the context of a reflective dialogue. This structure offered participants the opportunity to reflect on their role in the post observation tutorial and fore-grounded the ethnographic element of the research, as it facilitated the promotion of researchers as participants in the process under investigation.

The codification of recorded transcripts drew upon the concept of speech act semantics which highlights the illocutionary intentions of the contributors (Austin 1962, Searle 1969). This incorporated the linguistic element within the overall approach to data analysis. The semantic groupings then formed the basis from which the various themes presented in the discussion emerged. Overall, this approach converges with our desire to employ a reflexive approach to data collection and analysis by 'bringing together tools of ethnomethodological and interactionist sociolinguistics with tools of ethnography' (Copland 2010: 179). As a result, we were able to accomplish what Heller and Martin-Jones (2001:12) describe as the production of 'detailed and nuanced descriptions of talk in the context in which it occurs'.

\section{Participants}

The participants in this study comprised of fourteen trainee teachers on a part-time Posgtraduate Certificate in Education programme into which The Diploma in Teaching in the Lifelong Sector award is incorporated (PGCE: DTLLS). This is a training programme leading to the award of the professional qualification which is a requirement for gaining the Qualified Teacher Status (QTLS) award for teachers in the post-compulsory Education sector in the UK. There are a number of commonalities amongst participants; all are employed in various further education colleges and perceive their attendance as mandatory, have a minimum of five years experience and have been involved in evaluative feedback, and all have a minimum of a level three qualification in their subject area. This classifies them as training to become dual professionals (IFL, 2007) seeking to gain pedagogical professionalism in addition to their subject area professionalism. There are differences in terms of age and ethnicity although all were within the age range of forty to fifty years. In terms of ethnicity, only one of the participants was black and particular attention was paid to the views expressed by this minority participant to see if there was a pattern of race-induced difference. This was, however, done surreptitiously, as the researchers were concerned that undue pressure might be placed on this participant to represent the views of BME trainees.

\section{Quantitative analysis of Findings}

The entries made in Table 1 below were developed following the codification of the various findings as reported in the questionnaire. As a result, the subheadings used; psychological, technical, time, data protection and liberty are used to represent various features as reported by the participants. For example, when some of the participants indicated that they saw the tension generated by the demand that 
assessment places on the use of digital recording for reflective practice through feedback as a barrier, this was codified as a psychological issue. Similarly, when some participants indicated that the fact that others might have access to something they considered personal, this was considered as a data protection and liberty issue.

Table 1 Problems of Digital Recording

\begin{tabular}{|l|c|c|c|c|}
\hline & Frequency & Percent & Valid Percent & $\begin{array}{c}\text { Cumulative } \\
\text { Percent }\end{array}$ \\
\hline Valid psychological & 2 & 14.3 & 14.3 & 14.3 \\
technical & 4 & 28.6 & 28.6 & 42.9 \\
time & 4 & 28.6 & 28.6 & 71.4 \\
data protection and liberty & 1 & 7.1 & 7.1 & 78.6 \\
all four & 1 & 7.1 & 7.1 & 85.7 \\
combination of 1, 2, and 3 & 2 & 14.3 & 14.3 & 100.0 \\
& & & & \\
\hline Total & 14 & 100.0 & 100.00 & \\
\hline
\end{tabular}

As indicated in Table 1, there were four distinct categories of responses. The four categories were psychological, technical, time and data protection. More importantly, however, there was a clear indication that for some of the participants, it was a combination of more than one of the categories. Also, there was a clear indication that while a significant percentage, $28.6 \%$ identified technicality as a major problem, the remaining participants, $71.4 \%$ identified other factors. This lends credence to one of the rationales for this study; the need to explore emotional as well as physical problems associated with the use of digital recordings in the facilitation of collaborative feedback in the self reflection process.

\section{Psychological}

$14.3 \%$ of the participants identified psychological factors as being a source of problem in their use of the digital recording device. Central to the views of these participants were the issues of assessment requirements and the leader- led relationship that had existed between them and their tutors. These issues were explored further in the focus group discussion and constitute a line of investigation in the exploration of qualitative data in this study.

\section{Technical}

A noticeable percentage of respondents indicated that the major problems they had were technical. $28.6 \%$ of participants held this view. The major issues indicated in their responses related to installing the digital recording and mastering how to use it. While this was an issue for a significant number of participants, it was also clear that this was something they saw as surmountable. It is therefore safe to argue that physical problems are more easily surmountable, than problems that are less physical. 


\section{Time}

Time was also reported as being a major issue for participants. The major issues were time for installation and mastery of digital recorders, time spent on reviewing the recordings, and time spent on revisiting the recordings with their tutors. A total of $28.6 \%$ of participants held this view. It could be argued that for participants who held this view, their erstwhile dependence on teacher-led forms of feedback which required little from trainees in the feedback process might be influential. While this obviously saves trainees substantial time, evidence from various sources suggest that such an approach does not facilitate the development of self reflection skills.

\section{Data protection and liberty}

Though representing a minority of opinion, there was evidence that data protection and liberty can be an issue. The data indicated that this factor constituted a problem for some participants in the use of digital recording. The learning point emerging from this is that it is important to explore trainees' views regarding this issue before integrating the use of digital recordings into any training programme.

\section{Combinations:}

A total of 3 , representing $21.4 \%$ of participants indicated that a combination of these factors constituted problems for them in their engagement with the use of digital recordings. One participant indicated that a combination of all four factors identified above could be seen as constituting problems in the use of digital recordings while 2 participants, $14.3 \%$ felt that it was a combination of the first three factors. There are two potential points emerging from this. First, the use of technological devices is not a simple one dimensional process and therefore requires substantial thought before it is introduced. The second point is that participants' views may be informed by more than one element spanning the entire process from gaining feedback to the engagement with reflection. Hence, there were really no set of isolated factors but a combination of factors which in the end were focused on the entire process. The varying views, therefore, created the opportunity to explore the use of digital recordings as a component of the feedback process rather than as an independent element on its own.

\section{Qualitative analysis of findings}

Discussion within the focus group provided a general overview in addition to the identification of a number of emerging themes in relation to possible inhibitors. Although participants' views suggested that the experience proved invaluable (Sowe and Ade-Ojo, 2011) as reflected in some participants' comments that; "I could have done this every week" (R1), "think all teachers should do it"(R1), "Amazing opportunity - very rarely do teachers get that opportunity (R4), there were responses that required further exploration in order to facilitate a fuller understanding of the factors inhibiting the tool's use in the feedback process. These are presented and discussed thematically below.

\section{Theme 1: Conflation with assessment and judgement}

Participants were concerned with the seeming conflation of feedback with assessment. In particular, participants highlighted the inherent contradiction of this conflation with the developmental nature of using this tool. The response below illustrates this position: "It has two purposes though; 'One for self evaluation and one for judgement"(R7). 


\section{Discussion}

This concern reiterates the tension presented in the framework for evaluative feedback between the improvement and accountability models of evaluative feedback. Although assessment is not essentially an element of accountability from the view point of employer/employee relationship, it is possible that trainees may see assessment as an activity that requires them to give an account of their stewardship. This inevitable tension is, therefore, understandably a potential source of psychological inhibition and echoes Copland, Ma and Mann (2009) who suggest that the incompatibility of the assessment and development role that exists in teaching practice observation and evaluative feedback can result in disquiet and tension. Similarly, Brandt (2008) and Copland, Ma and Mann (2009) highlight the possible differing expectations of the trainer and trainee in regard to the assessment role of the trainer and the more evaluative role of the trainee. One position that has emerged from this is the fact that the reported tension is likely to remain regardless of whether the feedback is teacher-led in a conventional teaching practice observation or a post feedback discussion after use of a technological device. In the context of using the digital recording facility, therefore, it becomes evident that as long as the teaching practice observation remains a part of assessment, assessment is likely to remain significant as a barrier to using the tool.

\section{Theme 2: Replication of the ethos and culture of performativity}

A second theme to emerge from the focus group discussion is the potential for the tool to reinforce an ethos of performativity (Ball, 2003, Kelchtermans, 2007b). Participants highlighted the fact that using digital recordings offers the opportunity for entrenching the the-day- to- day climate of performativity in which professional teachers find themselves because of the element of permanence and continuity that it could potentially attract. The driver for this view is the opportunity for judgement which it readily provides. Typifying this position is a comment from one participant: "Is it possible to work against you?..."(R8).

\section{Discussion}

Participants' allusion to the possibility of 'judgement' that goes with the viewing of their practice must be seen in the context of their powerful perception of the situation. A pertinent question arises here: could 'judgement' be an indication of the possible vulnerability trainees feel in this situation particularly when the data for judgement is held on record? This brings a complexity to the situation by linking potential tutor assessment and 'judgement' to that of another theme of data protection of the trainee in regards to production of information that is often linked to the monitoring systems of an organisation (Ball, 2003). In our view, this segment of response brings to life the prevalent atmosphere and culture of performativity in the teaching profession (Ball, 2007 and Keltchermans, 2007). Emerging from this is a salient question for teacher educators; Is the environment of teacher education a total replication of the work environment? While we acknowledge the need to familiarise trainees with the environments that are similar to the one in which they are ultimately going to be employed, perhaps this needs to be tempered. What this research suggests is that the prevalent workplace culture appears to be so all-pervasive that even trainee teachers feel the impact. 


\section{Theme 3: Data protection and liberty}

Although expressed by a minority, there is an indication that data protection and liberty can be an issue. Comments by R8 that, "It's on record, Works against you for quality or not" highlights the issues of mistrust, access to personal data and the classical notion of liberty as very important for trainees in the context of the use of digital recordings.

\section{Discusson}

Halter (2004:9) reminds us that the use of technical devices such as digital recordings offers a permanent and accurate record. Further discussions with participants suggest that the element of permanence might well be an issue. In the age of what can be perceived as the dominance and all-pervasiveness of performativity (Ball, 2003, Kelchtermans, 2007b), the element of mistrust becomes inevitable. Although the recorded trend of performativity is often in relation to managers and the managed, the emergence of trust as an issue suggests that this phenomenon might be replicated in the context of trainees/tutors and trainers. The element of performativity would seem to be applied in this case to the data that the use of digital recording of trainees make available. For participants who expressed some form of apprehension in this context, there is a reasonable assumption that they might be judged in some way through the data made available and that they as trainees can be held accountable for it at a later date. Based on the foregoing, it is logical to assume that there is a possibility for the emergence of a level of mistrust between tutors and trainees on the one hand, and trainees and recorded evidence on the other.

Furthering the discussion on accountability and trust, O'Neill (2002) suggests that in a culture of accountability, trust is likely to be diminished. In this context, therefore, the use of technological devices, if not handled sensitively, may present a feeling of surveillance when located within the framework of a culture of accountability. In this situation, a trainee may feel an 'object of surveillance' (Matthews, 2006) with increased vulnerability (Kelchtermans, 2005, Bullough, 2005) and intimidation (Halter, 2004). The result, one can envisage, is a resultant reluctance to use such a device. As is the case with teachers, trainees' feelings of vulnerability are likely to be sharpened. A potential result of this situation is the acceptance of Kelchtermans' (2005) argument that vulnerability has in effect become a structural characteristic of the profession. What this research suggests is that vulnerability might well have become a structural characteristic of training in much the same way.

\section{Theme 4: Psychological impact on trainees' learners}

A particularly interesting theme to emerge from participants was the potential psychological impact that the use of digital recordings might have on trainees' learners. This potential inhibition was echoed throughout the focus group discussions. Indicative of this concern were additional comments such as; "Students might not agree to the filming", "Students can feel uncomfortable being filmed", "Students can be distracted by camera", "Some students do not feel comfortable to being filmed every single lesson".

\section{Discussion}

When explored during the focus group discussion, there was a strong indication that the main driver for the emergence of this theme is the fact that just as trainee teachers have a sense of responsibility for their own 'well being', they have the same sense of responsibility for their own learners' well being. This may come as no surprise, as it is arguably a reflection of professionalism on the part of these trainee teachers. 
Bearing in mind that the provision of a safe environment and promotion of the well being of those we teach is paramount as an aspect of teachers' professional characteristics and a crucial element of the lifelong learning sector's (LLS) professional standards, it should not come as a surprise that these trainee teachers, all of whom are already experienced teachers, place such emphasis on the comfort of their learners.

\section{Theme 5: Indirect Psychological factor}

Another emergent theme can be classified as being indirectly related to the psychological, as it draws on both the physical and psychological. Some participants suggested that some aspects of their perceived technical problems may also induce psychological barriers. This relates to comments on the limitations of the recording to capture all they do and, therefore, becoming off-putting for them. Comments such as, "Design of the classroom as small gap to capture just a few students and not the whole class"(R3), "somewhat flat on video', You do not get the feel of the class"(R8), and "Possible technical limitations with the camera not capturing all the action and interaction in the classroom" illustrate this position. The key issue here, in our view, is the fact that there might be layers to the sources and impact of significant factors in this context. It is, therefore, crucial that teacher educators do not take things at face value and that they draw on their reflective skills to drill down to the root in addressing some of these factors.

\section{Discussion}

Comments and opinions presented above may provide an insight into the importance that trainee teachers may place on ensuring that the viewer sees them for what they are. This need to be 'seen' which could possibly be accounted for in the context of the perception of the self could understandably metamorphose into a limitation of the device. This becomes significant because it has the potential to cause concern for trainees. In a way, it might again be seen as an expression of some form of residual apprehension which emanates from the notion of being judged and wanting to be represented in as best a light as possible. This position was further reiterated through responses such as, "I did not feel the observer was able to capture all aspects/elements of the lesson from viewing randomly selected snippets of the video of my lesson and therefore felt dissatisfied that they were commenting from a fully informed position". Although it might be argued that it was the trainee's choice that not all the lesson was viewed, this comment does highlight the high level of dissatisfaction a trainee may have if the opportunity to view them 'in total' is compromised.

Trainee teachers' sense of efficacy is often noted as very high on their priority list. The need for others to 'see that' is strong and may well relate to the issue of 'judgement and assessment' with trainees concerned that a judgement will be made without the full picture. In our view, this again echoes the conflicting conceptual realities of evaluative feedback as an instrument of accountability as against its conception as an instrument of development (OECD, 2009). In a similar iteration, this position underscores the observation that a negative public judgement may have a devastating effect (Kelchtermans, 1996, 1999, 2005). More importantly, it exemplifies how inhibitions in this context might overlap in terms of their impact. 


\section{Theme 6: Further technical problems}

Further technical problems were highlighted in relation to the quality of the recording in the postobservation evaluation: "the audio was a bit of a problem at times, e.g. when working together you can't pick out voices - just hearing mumbling and you can't tell if talking about work" (R4), "Basic camera set up not of a high enough quality for group work as much interaction can be missed'.

\section{Discussion}

At the heart of this was an issue around the quality of the digital recording devices. This brings into the equation cost which is directly responsible for the quality of the devices to be used in such endeavours. It is important, therefore, that cost should be considered as a potential inhibitor and that even when sufficiently available, careful consideration should be taken with the purchase of the technological device , ensuring in particular that it remains 'user friendly' for teachers. This factor was again brought into focus through trainees' comments in relation to the time commitment of the process and use of camera. Some participants noted, "Effective setting up of equipment is time consuming and requires specialist knowledge", "Setting up the video camera would require the teacher to spend some time with device and, therefore, teacher's attention would shift from the class to the device". While the latter comment reinforces the desire for the teacher to ensure greater efficacy, it also highlights the potential technical problems that using the device might contribute through a reduction in the desired self efficacy.

\section{Theme 7: Reiteration of findings from quantitative data}

One emergent theme that essentially reiterated the finding from quantitative data was the potential for inhibitions deriving from technicalities involved in the use of digital recordings. While the emergence of this inhibition might be understandable from the position that many trainees engaged with the installation and use of the digital device on their own and for the first, the time-related element of this problem may be surmountable and merely raises the importance of giving teacher trainees support in terms of training before they engage with the use of such devices. Indeed, it might actually provide a rationale for the integration of elements of ICT in the teacher training curriculum.

Related to the issue of technology was time. Apart from its association with the technical requirements of using the digital recording device, participants gave an indication that time as a variable can become a source of inhibition in using the device. Reflecting such concerns were comments like, "Time consuming. Find camera, get permission, watch it once done, watch it again with tutor etc. etc." , "The self-reflective process after the lesson", "The accompanying paperwork was too extensive, cumbersome and time consuming to complete...". This suggests that although the experience is seen as positive and enhanced by the inclusion of an evaluation framework document (Sowe and Ade-Ojo, 2011), the additional time required may inhibit its effective use, particularly in the backdrop of 'the busy teacher'.

\section{Conclusions}

This study sought to identify the crucial inhibitors to using a technological device as an instrument for promoting a more collaborative feedback process. The discussions around the findings of this project have highlighted the complexities of the situation. One interesting pattern that has emerged is the potential for one inhibitor to induce or relate to another consideration. For example, as demonstrated in the case of technical inhibitions, there was evidence that this might culminate in inducing a psychological inhibition. 
Also, there was evidence that psychologically grounded inhibitors might impact across different groups of stakeholders, as was the case with the psychological manifestation of the potential psychological aversion to being recorded which could cut across both trainee teachers and the students they teach. Furthermore, the development of a sense of vulnerability by trainee teachers, induced by the feeling that there is a permanent record of the activity, is a real consideration. This appears to resonate with the arguments of Ball (2005) on the prevalence of a climate of accountability and performativity in situations where data collection and monitoring is frequent.

A viable conclusion from this is that there is a need for a form of cast-iron guarantee to trainees in terms of the use and security of recordings. In our view, this might imply that a form of senior management level involvement might be necessary in order to assuage the anxiety that trainees might potentially feel if the use of digital recording is to be integrated into teacher education programmes. This may go some way to diminish the feeling of vulnerability and in turn, increase the trust that trainees hold for its use. It appears from this study that this will more likely induce trainees to willingly become a part of a process of a more collaborative feedback and echoes the argument of Mill (1992:19) that observations must take place "in an atmosphere of mutual trust and respect, without feelings of criticism or threat". This, in essence, will engender a perception of the use of technological devices as a communication and developmental tool rather than as a surveillance tool (Dyke, Harding, Lajeunesse, 2006).

Another issue emerging from this study revolves around assessment in teacher education. Whilst assessment remains an integral part of teaching observation in initial teacher training, the findings of this study suggest that it constitutes a limit to the extent to which trainees benefit from post-observation feedback and that the associated levels of anxieties for trainees will be difficult to allay. In our view, it may be necessary for teacher education providers to explore an alternative approach which will create a framework that separates post-observation feedback from the assessment strand of teacher education. Put in another way, the conceptualised purpose of evaluative feedback must be clearly understood and must recognise the difference between the accountability and developmental models of using it.

Engagement with technology was also a clear area of concern. It was clear that this has to do with both the perception of self and the element of apprehension, which can also be linked to the role of assessment. While the discussion above appears to have engaged with the assessment-related issue, it is important that the purely technical aspect must not be overlooked and be given considerable thought. Self-image and how one is perceived by others (Kelchtermans, 2005) appears to be a critical aspect for consideration in the development of a framework. While we do not have a ready-made solution to this issue here, it is important that teacher educators have an awareness of the potential inhibition that this factor might cause. At the least, teacher educators need to be aware of the capabilities of the instrument they opt to use, as well as the technical capabilities of their trainees.

Time is perhaps the most crucial issue here as this may be the most intransigent of the problems identified in this study. The key may lie in the assurance that the process, though time consuming, is overwhelmingly useful to the trainee. The need for an evaluative framework is paramount (Sowe and Ade-Ojo, 2011) although it is time consuming. This certainly calls on programme designers in teacher education to ensure that their evaluative frameworks is not unnecessarily time consuming. More importantly, it is essential that the benefit of using such a device is very evident to the trainee in terms of their professional development such that they might be considered as having benefits that outweigh their down-side. 
Finally, there is abundant evidence that a technology enhanced feedback process can be of tremendous use in the development of trainees. This, in our view, should not be limited to teacher-trainees/ trainers relationship alone. Given that feedback is an integral part of reflective practice and professional development, there is no doubt that any instrument that enhances its delivery will be of benefit in any professional field (e.g. nursing, business or lecturer development). The challenge for those who might want to enhance the effectiveness of feedback in professional development in other professional fields is to de-conflate it from its role as an assessment, monitoring and surveillance tool. As shown in this research, the conflation of a digital recording-enhanced feedback as an instrument for professional development and its use as an element of assessment has tended to limit its effectiveness. In order to fully benefit from its enhanced use as an instrument for professional development, therefore, it is important that professionals de-conflate its use from assessment.

\section{References}

Ade-Ojo. G. (2011). Beyond locutionary denotations: expanding trust between practitioners and policy. Research in Post-Compulsory Education Vol 16, No 2, 263-274.

Ade-Ojo, G.O. and Sowe, N. (2011). Using technology in the development of a collaborative approach to feedback and more active reflection: An exploration of trainee teachers' views. Procedia - Social and Behavioral Sciences, 29. pp. 503-519.

Alexander, R. (2005). Culture, dialogue and learning: Notes on an emerging pedagogy. Keynote Presentation given at the 10th International Conference of the International Association for Cognitive Education and Psychology. Durham, UK.

Austin, J. L. (1962). How to do things with words. Oxford: Oxford University Press.

Avalos, B. and J. Assael (2006). "Moving from resistance to agreement: The case of the Chilean teacher performance evaluation", International Journal of Educational Research, Vol. 45, No. 4-5, pp. 254-266.

Ball, S. (2003). The teacher's soul and the terrors of performativity. Journal of Educational Policy Vol 18, No 2, 215-228.

Bell, J. (2006). Doing Your Research Project: A Guide for First-Time Researchers in Education, Health and Social Science. Open University Press.

Brandt, C. (2008). Integrating feedback and reflection in teacher preparation. ELT J (2008) 62 (1): 37-46.

Bullough, R. V. (2005). Teacher Vulnerability and Teachability: A case study of a mentor and two interns. Teacher Education Quarterly. 23-39.

Coghlan, D., Brannick, T. (2010). Doing Action Research in your own organisation. London: Sage Publications Ltd.

Copland, F (2008). Deconstructing the discourse: Understanding the feedback event. In Sue Garton and Keith Richards, Professional encounters in TESOL, 5-23. London: Palgrave.

Copland, F., Ma, G. and Mann, S. (2009). Reflecting In And On Post-Observation Feedback In Initial Teacher Training On Certificate Courses. ELTED Vol. 12 Winter 2009, 14-22. 
Copland, F. (2010). Causes of Tension in post-observation feedback in pre-service teacher training: An alternative view. Teacher and Teacher Education 26, 466-472.

Cresswell, J. W. (2003). Research Design: Qualitative, Quantitative and Mixed Methods Approaches (2nd edn.) Thousand Oaks, CA: Sage.

Creese, A. (2008). Linguistic ethnography, in A. Creese, P. Martin, and N. Hornberger (Eds.) Encyclopaedia of Language and Education, Vol. 9. Springer.

Crow, G. and Wiles, R. (2008). Managing anonymity and confidentiality in social research: the case of visual data in Community research. NCRM Working Paper. ESRC National Centre for Research Methods. (Unpublished). [Online]. Available at: http://eprints.ncrm.ac.uk/459/ [Accessed 01/09/11].

Denscombe, M. (1998). The Good Research Guide for small-scale social research projects.

Buckingham: Open University Press.

Dymond, S. K. \& Bentz, J. L. (2006). Using digital videos to enhance teacher preparation. Teacher Education and Special Education, 29(2), 98-112.

Dyke, Harding and Lajeunesse, (2006). The use of synchronous digital video for observation of, and feedback on, teaching performance in the learning and skills sector. A report for the standard unit of the Department for Education and Skills: ITT Reform Pilots.

Edge, J. (2005). Continuing Cooperative Development. US: The University of Michigan Press.

Halter C (2004). Through the Lens: Digital Video Inquiry in Teacher Education. Two-year paper, TEP260C SPR04. [Online]. Available at: http://tepserver.ucsd.edu/ jlevin/tep-eScholarship/ThroughLens_Halter.pdf [Accessed 01/09/11].

Heller, M. And Martin-Jones, M. (2001). Introduction: symbolic domination, education and linguistic difference. In M. Heller and M. Martin-Jones (Eds.) Voices of authority: education and linguistic difference. Westport, CT: Ablex.

Hobbs, V. and Kubanyiova, M. (2008). 'The challenges of researching language teachers: What research manuals don't tell us' Language Teaching Research 2008 12: 495.

Kelchtermans, G. (2007b). Professional commitment beyond contract. Teachers' self-understanding, vulnerability and reflection. In J. Butcher and L. McDonald (Eds). Making a difference: Challenges for teachers, teaching, and teacher education (pp35-53). Rotterdam: Sense Publishers.

Kelchtermans, G (2005). Teachers' emotions in educational reforms: Self-understanding, vulnerable commitment and micropolitical literacy. Teaching and Teacher Education, 21, 995-1006.

Kelchtermans, G. (1996). Teacher vulnerability. Understanding its moral and political roots. Cambridge Journal of Education, 26(3), 307-323.

Kelchtermans, G (1999). Teacher career: between burnout and fading away? Reflections from a narrative and biographical perspective. In R Vandenberghe and M Huberman (Eds) Understanding and Preventing Teacher Burnout. A Sourcebook of International Research and Practice (pp.176-191). Cambridge: 
Cambridge University Press.

Kong, S., Shroff, R. and Hung, H. (2009). A web enabled video system for self reflection by student teachers using a guiding framework. Australian Journal of Educational Technology, 25(4), 544-558.

Matthews, B. (2006). Engaging Education Developing Emotional Literacy, Equity and Co-education. Maidenhead: Open University Press.

Millis, B. J. (2005,). Conducting effective classroom observations. Paper presented the 30th annual meeting of the Professional and Organizational Development Network, Milwaukee, MI. [Online]. Available at: http://www.txwescetl.com/services/teaching-observations/ [Accessed 01/09/11].

Mula, J. (2009). Going for $100 \%$ digital teaching and learning environment in accounting: the agony and the ectasy. In 2009 accounting and finance association of Australia and New Zealand Conference (AFAANZ), 04-07, July 2009 Adelaide, Australia.

OECD (2009a), OECD Review on Evaluation and Assessment for Improving School Outcomes: Design and Implementation Plan for the Review, OECD, Paris [OLIS Document EDU/EDPC(2009)3/REV1].

OECD (2009b), Creating Effective Teaching and Learning Environments: First Results from TALIS, OECD, Paris.

OECD (2009c) Teacher Evaluation: A Conceptual Framework and examples of Country Practices OECD-Mexico Workshop Towards a Teacher Evaluation Framework in Mexico: International Practices, Criteria and Mechanisms, held in Mexico City on 1-2 December 2009.

O'Neill, O. (2002). A Question of Trust. Reith Lectures 2002. [Online]. Available at: http://www.bbc.co.uk/radio4/reith2002/ [Accessed 01/09/11].

Punch, K. (2003). Introduction to Social Research: Quantitative and Qualitative Approaches. London: Sage Publications.

Rampton, B.(2007). 'Neo-Hymesian linguistic ethnography in the UK' Journal of Sociolinguistics, 11 (5), pp. 584-607.

Research for Teachers (2007). Using video as a tool for self-reflection. Research for Teachers.

Robinson, L. and Kelley, B. (2007). Developing reflective thoughts in preservice educators: utilizing role plays and digital video. Journal of Special Education Technology, 22(2), 31-43.

Searle, J. R. (1969). Speech Acts: An essay in the philosophy of language. Cambridge: Cambridge University Press.

Sherin, M. G. and Van Es, E. A. (2003). A new lens on teaching: learning to notice. Mathematics teaching in the middle school, 9(2), 92-95.

Stronge, J. and Tucker, P. (2003). Handbook on Teacher Evaluation: Assessing and Improving Performance, Eye On Education Publications. 
Tusting, K. and Maybin, J. (2007). Linguistic ethnography and interdisciplinarity: opening discussion.

Journal of Sociolinguistics, 11 (5), pp 575-583.

\section{Author Biographies}

Gordon Ade-Ojo is a principal lecturer in the School of Education and contributes to the lifelong learning teacher education and Postgraduate programmes. Gordon has authored books and published several articles in peer reviewed journals. Gordon is an academic associate of UNISA.

Nicola Sowe is an education consultant and researcher and contributes to the LLTE programme of the University of Greenwich. Nicola is particularly interested in the areas of curriculum development and student learning. 